Copyright (C 2008 IEEE. Reprinted from IEEE Sensors Journal, 2007; 7 (3):342-343

This material is posted here with permission of the IEEE. Such permission of the IEEE does not in any way imply IEEE endorsement of any of the University of Adelaide's products or services. Internal or personal use of this material is permitted. However, permission to reprint/republish this material for advertising or promotional purposes or for creating new collective works for resale or redistribution must be obtained from the IEEE by writing to pubs-permissions@ieee.org.

By choosing to view this document, you agree to all provisions of the copyright laws protecting it. 


\title{
2-D Wavelet Segmentation in 3-D T-Ray Tomography
}

\author{
Xiaoxia Yin, Student Member, IEEE, Brian W.-H. Ng, Member, IEEE, Bradley Ferguson, Member, IEEE, \\ Samuel P. Mickan, Member, IEEE, and Derek Abbott, Fellow, IEEE
}

\begin{abstract}
In this letter, segmentation techniques for terahertz (T-ray) computed tomographic (CT) imaging [1] are investigated. A set of linear image fusion and novel wavelet scale correlation segmentation techniques is adopted to achieve material discrimination within a 3-D object. The methods are applied to a T-ray CT image dataset taken from a plastic vial containing a plastic tube. This setup simulates the imaging of a simple nested organic structure, which provides an indication of the potential for using T-ray CT imaging to achieve T-ray pulsed signal classification of heterogeneous layers.
\end{abstract}

Index Terms-Tomography, T-rays, wavelet transform.

\section{INTRODUCTION}

$\mathbf{T}$ -RAYS is a collective term to describe the part of the electromagnetic spectrum from 0.1 to $10 \mathrm{THz}$. The application of T-rays, especially in the biomedical and security fields, is attractive owing to two intrinsic properties: a nonionizing nature and the ability to penetrate dry, nonpolar, and nonmetallic materials. Rapid improvements in T-ray detectors and sources make it possible to image objects through optically opaque layers. At present, 3-D T-ray CT imaging is being developed based on T-ray spectroscopy [1]. T-ray CT imaging has the promise to play an important role in a large number of clinical applications, particularly as a means of assisting clinical diagnosis. In this letter, a 3-D classification scheme to implement T-ray CT imaging is investigated. Image fusion and segmentation techniques, including a novel wavelet scale correlation method, are adopted to achieve the discrimination of heterogeneous materials within a 3-D object. The methods are applied to 4-D T-ray imaging datasets of a vial containing a tube, illustrated in Fig. 1, with an arrow line indicating the T-ray measurement path.

\section{BRIEF REVIEW TO TERAHERTZ IMAGING}

In T-ray CT, 4-D datasets are acquired. The axes of these datasets correspond to 1) the projection angle; 2 ) the offset, a perpendicular distance of the projection path to the rotation axis; 3 ) the vertical axis, perpendicular to the paper to label target heights; and 4) the sampled time. Two processing steps are required for T-ray CT reconstructions: i) 1-D Fourier transforms (FTs) of incident T-ray pulses; ii) filtered back projection of spatial FTs,

Manuscript received July 6, 2006; revised September 15, 2006; accepted November 27, 2006. The associate editor coordinating the review of this paper and approving it for publication was Prof. I. Barsony.

X. Yin, B. W.-H. Ng, S. P. Mickan, and D. Abbott are with the Centre for Biomedical Engineering and School of Electrical and Electronic Engineering, The University of Adelaide, SA 5005, Australia (e-mail: xxyin@eleceng.adelaide.edu.au; bwng@eleceng.adelaide.edu.au; spmickan@augean.eleceng.adelaide.edu.au; dabbott@eleceng.adelaide.edu.au).

B. Ferguson is with the Centre for Biomedical Engineering and School of Electrical and Electronic Engineering, The University of Adelaide, SA 5005, Australia, and also with the Tenix-Electronic Systems Division, Technology Park, Mawson Lakes, SA 5095, Australia (e-mail: brad.ferguson@tenix.com).

Color versions of one or more of the figures in this paper are available online at http://ieeexplore.ieee.org.

Digital Object Identifier 10.1109/JSEN.2006.890159

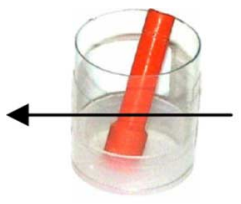

(a)

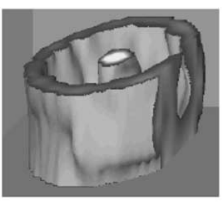

(b)

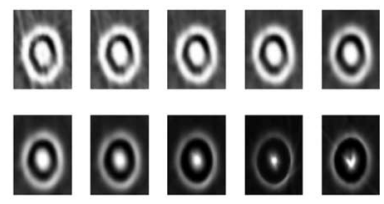

(c)
Fig. 1. Reconstructed T-ray CT. (a) Target object photograph with arrow line indicating the measurement height of $7 \mathrm{~mm}$. (b) Reconstructed 3-D T-ray CT. (c) Reconstructed T-ray CT slides at the first ten frequencies, in increasing order from top left at the object height of $7 \mathrm{~mm}$.

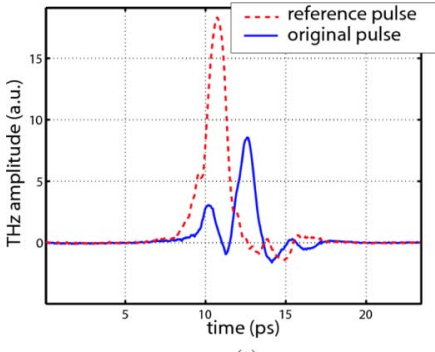

(a)

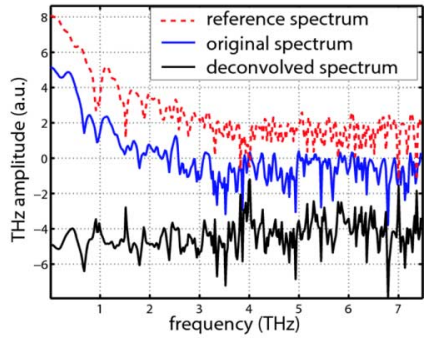

(b)
Fig. 2. (a) Detected T-ray signal and reference; (b) their spectra in log scale with offsets of 2 and 4 a.u. corresponding to the original and deconvolved spectra, respectively, for clarity.

which is a collection of 1-D projections at all projection angles. The processing used in this T-ray CT experiment are described in detail in [1]. Fig. 1(a) shows an optical photograph of the experimental setup. Vertical slices used for this experiment are spaced $1 \mathrm{~mm}$ apart, while each T-ray CT reconstructed slice image has a resolution of $89 \times 89$ pixels. Fig. 1 (b) shows the full reconstructed 3-D T-ray CT model; Fig. 1(c) shows the reconstructed 2-D T-ray CT slices regarding the 10 lowest frequencies at a height of $7 \mathrm{~mm}$.

Fig. 2(a) shows a detected T-ray signal and its reference pulse. Fig. 2(b) shows magnitudes of Fourier transform of pulses in (a) with an offset for clarity. The black solid line is the deconvolved $\mathrm{T}$-ray spectrum. The oscillations that appear in the incident T-ray pulses and as dips on the T-ray spectra are a result of water vapor [3], [4] in the beam path. From the spectra, it is evident that the useable bandwidth of the signals is limited to $2 \mathrm{THz}$, due to the reduction in signal strength as frequency increases. In addition, there are a large number of noise sources in a T-ray system, which are discussed in [5]. As a consequence, the SNR of the T-ray signals are high for only the lower parts of the frequency range.

\section{Methodology}

The setup consisting of a tube inserted inside a vial is imaged at various heights, ranging from 5 to $9 \mathrm{~mm}$ (from the bottom), in 1-mm increments. To achieve 3-D T-ray CT classification, image fusion methods and segmentation techniques are applied to extract three different target segments. The image fusion is achieved through merging two or more images at the same target height. A linear combination of weighted slice images is computed for image fusion with an aim to achieve border consistency 
in the fused T-ray CT images. Due to SNR limitations in the experimental apparatus at high frequencies discussed above, only images corresponding to the ten lowest frequencies, from 0.0213 to $0.213 \mathrm{THz}$, are selected. In this work, the weighting scheme is empirically chosen to be $1 / 15$ for the five lower frequencies and $2 / 15$ for the five higher frequencies to compensate for the attenuation in signal strength in the higher frequency components. The resultant fused image has clear target contours and shows strong contrast between the target regions and the background.

Segment detection achieves differentiated subdivision of constituent regions of an image. In this letter, a novel 2-D wavelet scale correlation method is used to extract target segments from the background. This method is motivated by 1-D wavelet scale correlation denoising. First, the target cross sections are assumed to be corrupted by additive white Gaussian noise, which is distributed randomly. The target objects are separated by their absorption coefficients, which are indicated by the intensity in the images. With the incremental wavelet scale, the noise is reduced and the target intensity (energy) is increased in an image. After computing undecimated 2-D DWTs [6], [7] of fused T-ray CT images, an increased energy with an increase in wavelet scale is used as a cue to extract the target regions. The wavelet scale correlation based segmentation algorithm is summarized as follows.

1) Calculate undecimated 2-D DWT coefficients at first and second scales: $s_{1}(m, n), s_{2}(m, n)$, respectively.

2) Compute the correlation $R_{1,2}(m, n)$ for the two scales

$$
R_{1,2}(m, n)=s_{1}(m, n) \times s_{2}(m, n) .
$$

3) Compute the energy of $R_{1,2}(m, n)$ and $s_{1}(m, n)$ and use them to normalize the correlation coefficients

$$
\begin{aligned}
E_{s 1} & =\Sigma s_{1}^{2}(m, n), \quad E_{R 1,2}=\Sigma R_{1,2}^{2}(m, n) \\
R_{1,2}^{*}(m, n) & =R_{1,2}(m, n)\left(E_{s 1} / E_{R 1,2}\right)^{1 / 2 .}
\end{aligned}
$$

4) If $\left|R_{1,2}^{*}(m, n)\right|>\lambda\left|s_{1}(m, n)\right|$, $(\lambda$ is a parameter, chosen to be 1 for this experiment), the pixel at $(m, n)$ is extracted as part of a target segment, otherwise it is regarded as background.

The Canny edge detector, combined with Otsu's threshold method [2], is used to perform the final subtraction of each target function edge [2] in the T-ray CT image. The current algorithm only requires a single parameter $\lambda$ in the threshold.

\section{EXPERIMENTAL RESULTS}

The algorithm described in Section III is applied to extract segments corresponding to the glass vial and plastic tube. The extracted approximate subimages, after computation of the 2-D DWT of the $89 \times 89$ fused images, are shown in Fig. 3(a) and (b); the Daubechies 4 wavelet is used in this case. The result of the correlation is shown in Fig. 3(c). Fig. 4(a) illustrates the separated segment images corresponding to height from bottom to top equal to 5 to $9 \mathrm{~mm}$.

\section{Segmentation Quality}

The segmentation quality cannot be directly verified since the explicit ground truth is not known. This is typical of tomographic applications - the subjects are not cut up to provide knowledge of the ground truth. In this experiment, we exploit the fact that the internal structure, the tube, is straight. This im-

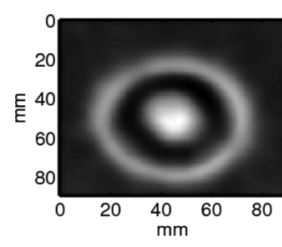

(a)

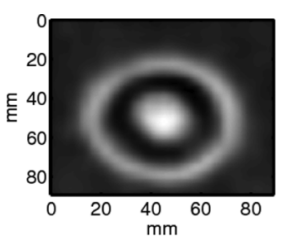

(b)

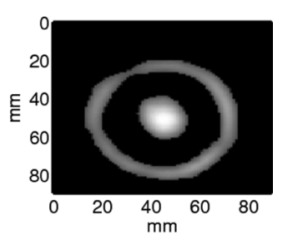

(c)
Fig. 3. Illustration of the approximate and correlated images after two scales of 2-D DWT of T-ray CT fused image. (a) The first-scale approximate image of 2-D DWT of T-ray CT image. (b) The second-scale image of 2-D DWT of T-ray CT image. (c) The correlation output image.

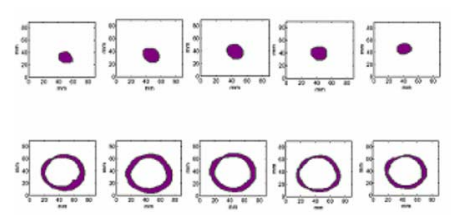

(a)

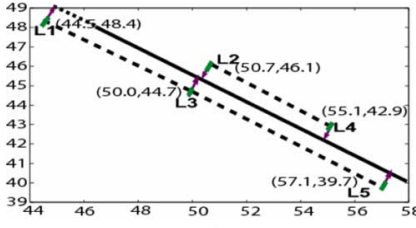

(b)
Fig. 4 (a) Segmented image sets at heights from 5 to $9 \mathrm{~mm}$, from bottom of vial. The upper subfigures are the tube segments; the lower subfigures are the vial segments. (b) Centroid locations of tube segment. The vertical and horizontal axes represent numbers of pixels.

plies that the segmented glass tube positions [see top row in Fig. 4(a)] should be proportionally displaced from each other, since they correspond to constant increments of $1 \mathrm{~mm}$ in height. To obtain this measurement, we find the centroid of the extracted glass tube for each height, L1-L5, and the resultant $x$ and $y$ locations are plotted in Fig. 4(b).

The linear regression line, with slope of -0.7 and an offset of 81.54 pixels, is used to fit the five measured centroids. The mean square error of each point to the given solid line is 1.034 . This indicates that our algorithm is capable of locating the centroid of the plastic tube to within a few pixels. Given the number of noise sources and the quality of the reconstructed images, this error is considered to be reasonable.

\section{CONCLUSION}

This letter investigates an application of wavelet based segmentation-by-fusion. It is a first exploration of automatic T-ray CT target identification within 3-D heterogeneous structures. The algorithm successfully segmented different target regions and was able to correctly locate the regions to within a few pixels.

\section{REFERENCES}

[1] B. Ferguson, S. H. Wang, D. Gray, D. Abbott, and X.-C. Zhang, "Towards functional 3D T-ray imaging," Phys. Med. Biol., vol. 47, no. 2002, pp. 3725-3742, 2002.

[2] R. C. Gonzalez and R. E. Woods, Digital Image Processing. Englewood Cliffs, NJ: Prentice-Hall, Inc., 2002.

[3] D. M. Mittleman, R. H. Jacobsen, and M. C. Muss, "T-ray imaging," IEEE J. Sel. Topics Quant. Electron., vol. 2, no. 3, pp. 679-692, Mar. 2002.

[4] S. Hadjiloucas, R. K. H. Galvão, and J. W. Bowen, "Analysis of spectroscopic measurements of leaf water content at terahertz frequencies using linear transforms," J. Opt. Soc. Amer. A, vol. 19, pp. 2495-2509, 2002.

[5] B. Ferguson and D. Abbott, "De-noising techniques for terahertz responses of biological samples," Microelectron. J., vol. 32, no. 2001, pp. 943-953, 2001.

[6] S. G. Mallat, A Wavelet Tour of Signal Processing. San Diego, CA Academic, 1999.

[7] R. Galvão, S. Hadjiloucas, J. Bowen, and C. Coelho, "Optimal discrimination and classification of THz spectra in the wavelet domain," Opt. Expr., vol. 11, pp. 1462-1473, 2003. 